\title{
COMPARATIVE ASSESSMENT OF VERY HIGH RESOLUTION SATELLITE AND AERIAL ORTHOIMAGERY
}

\author{
P. Agrafiotis, A. Georgopoulos \\ National Technical University of Athens, School of Rural and Surveying Engineering, Lab. of Photogrammetry \\ Zografou Campus, Heroon Polytechniou 9, 15780, Zografou, Athens, Greece
}

(pagraf,drag)@ central.ntua.gr

Commission I, WG I/4

KEY WORDS: Pleiades, Accuracy, Radiometry, Orthoimage, Statistical Analysis

\begin{abstract}
:
This paper aims to assess the accuracy and radiometric quality of orthorectified high resolution satellite imagery from Pleiades-1B satellites through a comparative evaluation of their quantitative and qualitative properties. A Pleiades-B1 stereopair of high resolution images taken in 2013, two adjacent GeoEye-1 stereopairs from 2011 and aerial orthomosaic (LSO) provided by NCMA S.A (Hellenic Cadastre) from 2007 have been used for the comparison tests. As control dataset orthomosaic from aerial imagery provided also by NCMA S.A $(0.25 \mathrm{~m}$ GSD) from 2012 was selected. The process for DSM and orthoimage production was performed using commercial digital photogrammetric workstations. The two resulting orthoimages and the aerial orthomosaic (LSO) were relatively and absolutely evaluated for their quantitative and qualitative properties. Test measurements were performed using the same check points in order to establish their accuracy both as far as the single point coordinates as well as their distances are concerned. Check points were distributed according to JRC Guidelines for Best Practice and Quality Checking of Ortho Imagery and NSSDA standards while areas with different terrain relief and land cover were also included. The tests performed were based also on JRC and NSSDA accuracy standards. Finally, tests were carried out in order to assess the radiometric quality of the orthoimagery. The results are presented with a statistical analysis and they are evaluated in order to present the merits and demerits of the imaging sensors involved for orthoimage production. The results also serve for a critical approach for the usability and cost efficiency of satellite imagery for the production of Large Scale Orthophotos.
\end{abstract}

\section{INTRODUCTION}

As satellite optical sensor technology progresses, very high resolution (VHR) images from space become available and lucrative for large scale mapping. Resolutions of less than $1 \mathrm{~m}$ reaching the level of a few tens of centimetres are common today thus enabling the users to distinguish fine detail on the earth's surface, like buildings, individual trees and even smaller objects. For applications concerned with mapping, cadastral recording and land monitoring VHR satellite imagery is directly challenging conventional or even digital aerial images of comparable resolution

\subsection{Motivation}

The Pléiades $1 \mathrm{~B}$ are a very high-resolution satellite constellation delivering 50-cm Ortho products as a standard (Astrium, 2012). In the context of the Pleiades evaluation program, the Laboratory of Photogrammetry of National Technical University of Athens acquired a triplet of high resolution images taken in 2013 by Pleiades 1B over the small Cyclades island of Antiparos in order to assess their usability and accuracy and compare it to similar satellite sensors. In the literature, the radiometric characteristics and the geometric accuracy of optical sensors and their resulting imagery have been extensively addressed in various contribution (Zhou and Li, 2000; Greenfeld, 2001; Niu et al., 2004; Eisenbeiss et al.,2004; Ioannidis and Katsigiannis, 2006; Cheng and Chaapel, 2008; Aguilar et al., 2008; Crespi and De Vendictis, 2009; Jacobsen, 2011). However, these contributions do not perform any comparative evaluation on the quantitative and qualitative properties of the resulting orthoimagery exploiting aerial Large Scale Orthophotos and Very Large Scale Orthophotos.

This paper aims to report the assessment of the accuracy and radiometric quality of orthorectified high resolution satellite imagery from Pleiades-1B satellites through a comparative evaluation of their quantitative and qualitative properties. In addition, the advantages and limits of the Pleiades Imaging for producing Large Scale Orthophotos (LSO) are investigated.

\section{DATASETS}

A Pleiades 1B stereopair of high resolution images taken in 2013 (0.50m GSD), two adjacent GeoEye-1 stereopairs $(0.50 \mathrm{~m}$ GSD) acquired for a diploma thesis (Ioannou and Georgopoulos, 2013) from which a high resolution orthomosaic had already been produced and aerial LSO (0.50m GSD) provided by the Hellenic National Cadastre \& Mapping Agency S.A. (NCMA S.A) from 2007 have been used for the comparison tests. As control dataset aerial orthomosaic (VLSO) was also provided also from NCMA S.A with $0.25 \mathrm{~m}$ GSD from 2012.

The study area, the small Cycladic island of Antiparos has an area of $35000 \mathrm{~km}^{2}$ and it is very close to Paros island. It measures $12.5 \mathrm{~km}$ in length and $5.5 \mathrm{~km}$ in width and has a coastal perimeter of $54 \mathrm{~km}$. Even though the island is almost flat, a few little hills in the centre reach a maximum height of 300 metres. The landscape is rather wild and varied including a main settlement and agricultural fields. Finally, on the west coast there are steep cliffs. 


\subsection{Test Dataset}

2.1.1 The Pleiades 1B Imagery: As already mentioned, the Pléiades twins are very high-resolution satellites delivering $50 \mathrm{~cm}$ Ortho products as a standard (Astrium, 2012). Pleiades1B satellite sensor was successfully launched on December 2, 2012. Built by AIRBUS Defence \& Space, the satellite was launched from a Soyuz launcher at the European Space Centre in French Guiana. Pleiades-1A and 1B satellites will be phased $180^{\circ}$ apart in the same near-polar sun-synchronous orbit at an altitude of $694 \mathrm{~km}$, enabling daily revisits to any location on the planet. The sensor can reach a ground resolution of $0.7 \mathrm{~m}$ in panchromatic mode and $2.8 \mathrm{~m}$ in multi-spectral mode in vertical direction. The images provided for this work were acquired in the tri-stereo mode for 3D information. According to this acquisition scheme, the satellite rotates around its axis and the HiRi camera scans a target area from three different viewing directions during one pass, thus resulting in a triplet (Gleyzes et al., 2012). However, for reasons of objectivity in comparison with data from GeoEye-1, only the 2 external images of the tristereo were used. The images were acquired on 9 April 2013 in the morning within 22.5 seconds. The average viewing angles of the two selected images are, respectively, $6.30^{\circ}$ and $9.47^{\circ}$ in across-track direction with respect to the nadir and $1.63^{\circ}$ and $12.39^{\circ}$ in across-track direction (Figure 1).

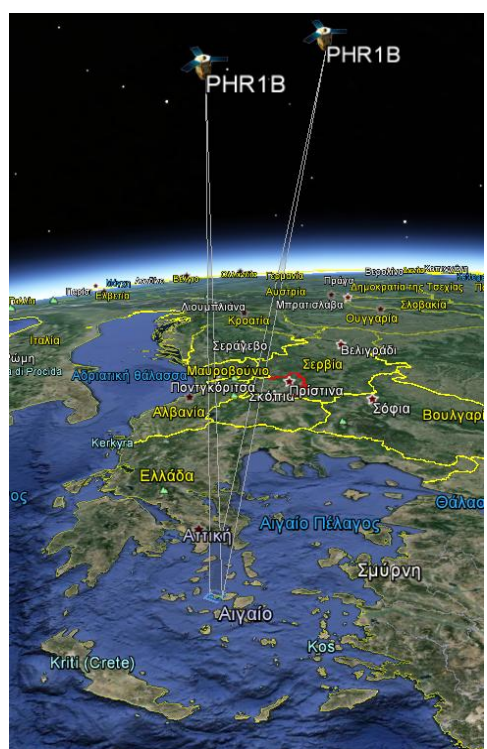

Figure 1. Stereo acquisition for 3D applications (Google Earth preview of the footprints and the satellite's position)

2.1.2 The GeoEye-1 Imagery: Geoeye-1 satellite was launched in September 2008. The sensor's vertical ground resolution of $0.41 \mathrm{~m}$ for panchromatic imagery and $1.65 \mathrm{~m}$ for the 4-band multispectral imagery is resampled to $0.50 \mathrm{~m}$ and $2.00 \mathrm{~m}$, respectively for commercial customers. The Geoeye-1 sensor was designed mainly for commercial use, with Google as one of its most valued customers. They use the imagery for their Google Maps and Google Earth applications. Geoeye Inc. markets this imagery by offering three basic product packages, which are distinguished by the level of positional accuracy they produce.

For the purposes of this study, two adjacent Geoeye-1 stereopairs from December 2011 were used. These high resolution stereopairs were collected in the same orbital pass, thus minimizing changes in lighting or scene content. They are accompanied by an RPC camera model file for georeferencing.
The images were collected during the single orbital pass and they consist of an image collected at a low elevation angle of above 60 degrees as well as an image collected at a high elevation angle of above 72 degrees. Choices of projection, including epipolar projections for stereo applications are also provided. The data consists of 8 or 11 bits per pixel imagery and is provided in GeoTIFF format, including metadata files.

2.1.3 The Aerial Imagery Orthomosaic (LSO): The tested LSO provided by NCMA S.A had $0.50 \mathrm{~m}$ GSD and the imagery were taken on 2007. This dataset has a geometric accuracy of RMSExy $\leq 1.41 \mathrm{~m}$ (95\% confidence level).

\subsection{Control Dataset}

In order to evaluate the produced orthoimages, accuracy specifications had to be set as a reference for the purpose of inferring about the actual metric efficiency of the product. As control dataset aerial imagery orthomosaic (VLSO) provided by Hellenic National Cadastre \& Mapping Agency S.A. (NCMA S.A) was used. It had $0.25 \mathrm{~m}$ GSD and the imagery was taken on August 12th 2012. According to NCMA S.A. the orthomosaic of the control dataset was created from aerial imagery of a scale of 1:15000 using a ZEISS RMK TOP 30 film camera having a lens of $153.12 \mathrm{~mm}$. To produce this orthoimagery, a DEM was created having $5 \mathrm{~m}$ grid interval.

\section{METHODOLOGY}

The methodology applied in this paper follows the well-known photogrammetric procedures in order to compare and assess the radiometric and geometric performance of very high resolution imagery from Pleiades $1 \mathrm{~B}$ and GeoEye-1 and aerial orthoimagery. Main goal of the methodology is to deliver reliable and objective results through a statistical analysis of the metric results exploiting commonly accepted standards for accuracy testing of orthoimages.

\subsection{GCP measurements and Distribution}

A number of ground control points were determined by GPS observations, which were suitably post-processed in order to get accurate measurements (mean uncertainty of $0.010 \mathrm{~m}$ ), distributed all over the area of interest. Some of these points have been used for the determination of orientation parameters as GCPs, and the rest as check points.

\subsection{Pansharpening}

The three stereopairs, 2 for GeoEye- 1 which contain the northern and southern regions of the island of Antiparos and one for Pleiades $1 \mathrm{~B}$ containing the whole island, included 5 bands; one panchromatic with a GSD of $0.50 \mathrm{~m}$ and four multispectral (R, B, G and NIR) with a GSD of $2.00 \mathrm{~m}$. For this study, only the three multispectral bands Red, Green and Blue were necessary.

Thus, pansharpening procedures took place in order to enable the further photogrammetric processing of the imagery. Various algorithms were tested in order to achieve the optimal visual result, including the Principal Component Analysis technique, the IHS (Intensity-Hue-Saturation), the Brovey fusion and the HCS (Hyperspherical Color Space) Resolution Merge. From these tests, the algorithm HCS Resolution Merge proved to yield the most acceptable visual results for the available Geoeye-1 stereo pairs and the algorithm IHS for Pleiades 1B stereopair. 
3.2.1 IHS for Pleiades 1B Data: A high-resolution colorized product from Pleiades 1B data was produced by using the IHS pan-sharpening algorithm. The Intensity-Hue-Saturation (IHS) method (Haydn et al., 1982) has been widely used (Carper et al., 1990, Chavez and J. Bowell, 1988, Edwards and P. Davis 1994, $\mathrm{Tu}$ et al., 2004) for pansharpening satellite images. In this frequently used method, a component derived from the MS image is substituted for a component derived from the PAN image and then the fused or pansharpened image is obtained from the inverse transformation.The basic idea is to first transform the MS image into intensity (I), hue $(\mathrm{H})$ and saturation (S) components (IHS colour space). The next step is to adjust the Pan image so that it has the same mean and variance as the intensity component of the MS image. The intensity component is then replaced with the appropriately scaled Pan image and finally the inverse IHS transformation is taken to get the fused image. The IHS method produces images that have high spatial resolution and low spectral quality (Palsson et al., 2012).

3.2.2 HCS for GeoEye-1 Data: Hyperspherical Color Space (HCS) pan-sharpening (Padwick et al, 2010) is an algorithm especially designed for processing images captured by the Worldview-2 satellite, which supports an 8 band multispectral and panchromatic sensor. The main concept of the algorithm is a transformation from the local color space to the hyperspherical color space. The multispectral RGB data are redefined by its band components into angular variables $(\varphi 1$, $\varphi 2, \varphi 3)$ which describe the color or hue, and a radial component (I) which defines the intensity of the color in hyperspherical space. The naïve approach of the algorithm simply replaces the multispectral intensity component with an intensity matched version of the panchromatic band using the normalized mean and standard deviation of the hyperspherical data. This is followed by a reverse transformation into the RGB color space for the compilation of the pan-sharpened image (Padwick et al, 2010). The technique is applicable to images composed of at least three bands and ideal for those with a great number of bands, such as Worldview-2, but the results were more than satisfactory for the Geoeye-1 RGB image of this project.

\subsection{Photogrammetric Georeference}

The photogrammetric georeference was performed using the commercial software LPS and Photomod. To this direction, after a 1st degree polynomial Rational Polynomial Coefficients (RPC) refinement, tie points and ground control points (GCPs) were measured on the images. 4 GCPs and 6 checkpoints were used for Pleiades 1B data achieving a RMSE of $0.045 \mathrm{~m}$ while 5 GCPs and 10 checkpoints were used for GeoEye-1 data achieving a RMSE of $0.160 \mathrm{~m}$.

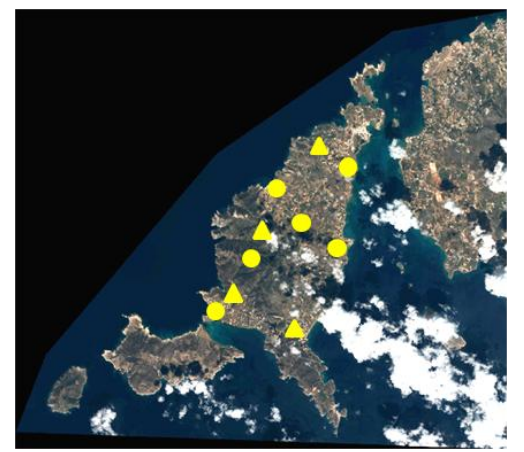

Figure 2. Ground control points and check points used for Pleiades 1B data
The occurred deviation between the RMSE of Pleiades 1B and GeoEye-1 data is justified by the increased radiometric saturation of GeoEye-1 imagery and is discussed further in Section 4.1. At this point it is noted that on the Pleiades 1B data less GCPs and checkpoints were measured because of the clouds on the lower right corner of the imagery.

\subsection{DEM Extraction and Orthoimage Production}

For the DSM extraction, an automatic procedure was applied for Pleiades 1B while a semi-automatic one was applied for the Geoeye-1 stereopair. The semi-automatic procedure was implemented because of problems occurring on coastal and rocky areas presenting steep terrain. In addition, GCPs were used for transferring the scale and real elevation values while the grid interval was selected as $10 \mathrm{~m}$.

As regards the production of the orthoimages (Figure 3,4), they were created using bilinear interpolation. A GSD of $0.50 \mathrm{~m}$ was selected while it should be mentioned that the orthorectification of all images of the stereopairs was performed in order to check the DSM for gross errors by subtracting them.

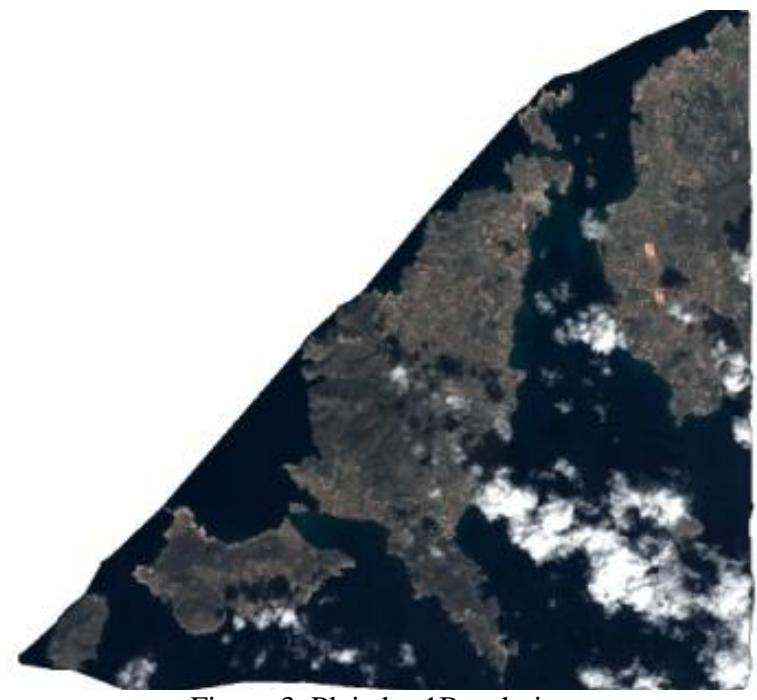

Figure 3. Pleiades 1B orthoimage

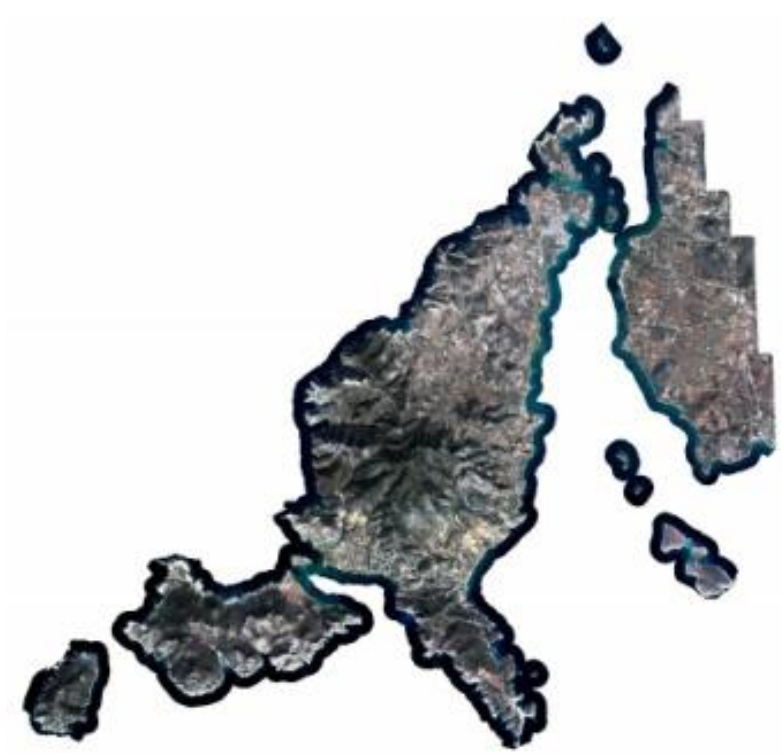

Figure 4. GeoEye-1 orthomosaic 


\section{QUALITY AND ACCURACY ASSESSMENT}

The results are presented with a statistical analysis and they are evaluated in order to present the merits and demerits of the imaging sensors involved. To this direction the Standard Deviation $(\sigma)$ or sigma are computed as an indicator of how well the measurements fit to each other and as a measure of precision. In addition, the Root Mean Square Error (RMSE) is computed for Northing and Easting coordinates. As observed, in aerial LSO and Geoeye-1 orthomosaic, the Standard Deviation and $\mathrm{RMSE}_{\mathrm{X}, \mathrm{Y}}$ differ, indicating a systematic error in $\mathrm{Y}$ axis. Thus, a bias removal procedure was applied in order to evaluate orthoimages objectively and accurately.

\subsection{Image Quality Assessment}

The radiometry of an image is satisfactory when the relationship between the ground reflectance of the target and the grey level of the pixel on the image is correct (Crespi and De Vendictis, 2009). Pleiades $1 \mathrm{~B}$ and GeoEye- 1 sensors provide images with radiometric resolution of $12 \mathrm{bit} / \mathrm{pixel}$. Thus, a higher dynamic range and signal-to-noise ratio with respect to traditional scanned 8-bit/pixel images is expected (Poli et al., 2014).

4.1.1 Visual Assessment: Visual inspection of the orthoimages was applied in order to identify errors and/or image defects. As an example, in GeoEye-1 images some abrupt changes of brightness and contrast were observed. In addition, many regions presented extremely dark/light tones, predominantly on the buildings and along the roads.
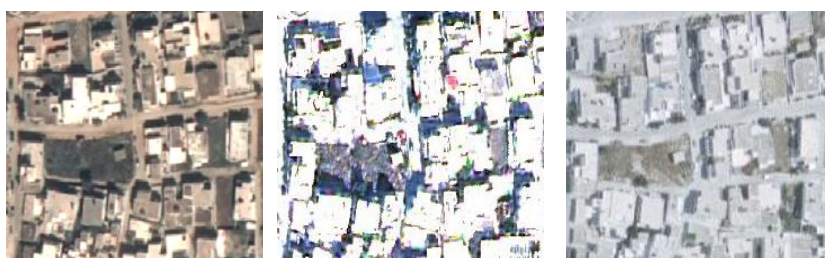

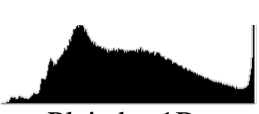

Pleiades 1B

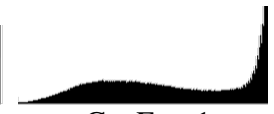

GeoEye-1

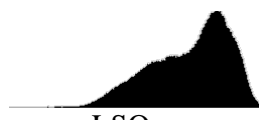

LSO
Figure 5. Examples of visual errors/defects of the orthoimages and their corresponding histograms
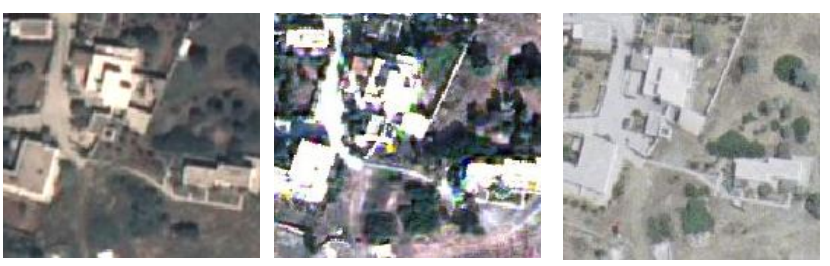

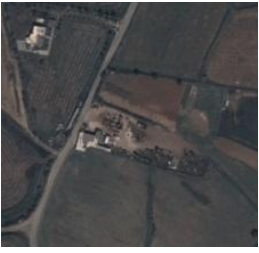

Pleiades 1B

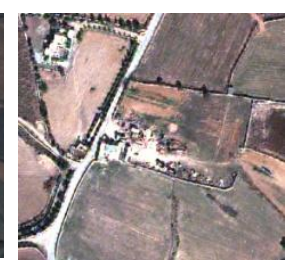

GeoEye-1

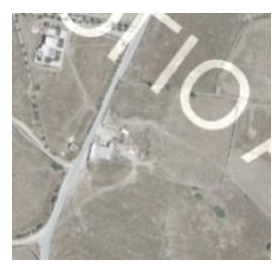

LSO
Figure 6. Examples of visual errors/defects of the orthoimages
Contrary, in Pleiades 1B image, the radiometric saturation of certain regions is especially prominent presenting much more information and clearer forms (Figure 5, 6). Moreover, the orthoimage resulting from Pleiades $1 \mathrm{~B}$ imagery is much darker than LSO and Geoeye-1 imagery. Important radiometric changes between two or more images are critical during automatic homologue point identification and during image correlation thus causing mismatches and wrong height estimation (Poli et al., 2014).

Histograms are the basis for numerous image processing techniques. In addition, histograms provide useful image statistics. In a dark image, the components of the histogram are concentrated on the lower side of the intensity scale while these components of a light image are biased toward the higher side of the scale. The histogram of a low contrast image has a limited range while on a high contrast image the components of the histogram cover a wide range of the intensity scale (Gonzalez and Woods, 2002). In figure 5, histograms of the presented part of the study area, indicates that LS Orthomosaic is characterized by low contrast and high luminosity. However, GeoEye-1 orthomosaic presents higher luminosity than LS Orthomosaic and very high frequency of tones near the white (255). Pleiades 1B orthoimagery presents a more normalized histogram with a wide range and components concentrated near the low side of the scale, indicating a darker image.

In addition to visual inspection of the images, the noise level and the geometrical resolution and sharpness which is described by the Modulation Transfer Function (MTF) are encountered in the literature. By the term noise the non-homogeneities in the image are described and it is evaluated on non-homogeneous areas. Over these areas, the noise variation is considered as function of intensity. It should be noted that noise for CCDimages is not additive but intensity-dependent (Poli et al., 2014). The Modulation Transfer Function (MTF) is used to estimate the spatial performance of an imaging sensor. In case of on-orbit MTF estimation, the edge method is generally used (Helder and Choi, 2003; Kohm, 2004; Leger et al., 2004; Crespi and De Vendictis, 2009, Poli et al., 2014).

\subsection{Spatial Accuracy assessment}

The two resulting orthoimages and the aerial imagery orthophoto (LSO) were evaluated relatively and absolutely for their quantitative and qualitative properties. Check points were measured on the VLSO product and test measurements were performed using the same check points in order to establish their accuracy both as far as the single point coordinates as well as their distances were concerned. 26 Check Points for Pleiades 1B and 26 Check Points for Geoeye-1 and LSO were distributed in total according to JRC Guidelines for Best Practice and Quality Checking of Ortho Imagery (Kapnias et al., 2008) and National Standard for Spatial Data Accuracy (NSSDA) (FGDC, 1998) standards while areas with different terrain relief and land cover were also included. Performed tests were also based on JRC and NSSDA accuracy standards.

4.2.1 The National Standard for Spatial Data Accuracy (NSSDA): The NSSDA implements a statistical and testing methodology for estimating the positional accuracy of points on maps and in digital geospatial data, with respect to georeferenced ground positions of higher accuracy. The NSSDA applies to georeferenced maps and digital geospatial data, in either raster, point, or vector format (FGDC, 1998). 
The NSSDA does not define threshold accuracy values and uses the root-mean-square error (RMSE) to estimate positional accuracy.

4.2.2 JRC Guidelines for Best Practice and Quality Checking of Ortho: These guidelines apply to digital orthoimagery products, generated from either film cameras or digital sensors, on both airborne or satellite platforms for the scope of applications requiring accuracy of $0.5 \mathrm{~m}-10 \mathrm{~m}$ pixel size and large scale mapping or cadastre applications $(0.5 \mathrm{~m}$ or better). All stages of the production chain affecting geometric accuracy and radiometric quality of the final product are considered (Kapnias et al., 2008). As with the NSSDA procedure, the final orthoimage is assessed using the RMSEx and RMSEy and the associated DEM from RMSEz. The use of RMSE provides a straight forward global statistic for assessing the final geometric accuracy. Additional indeces such as the mean error and the error standard deviation can be used in order to better describe the spatial variation of errors or to identify potential systematic discrepancies.

4.2.3 Checkpoints Selection and Distribution: According to the NSSDA and JRC Guidelines, accuracy testing should be performed using an independent source of higher positional accuracy. The accuracy of the independent test points should fall within one-third of the intended accuracy (95\% confidence level) of the examined dataset. A minimum of 20 well defined test points should be used to evaluate the accuracy of the dataset. The check points will be ideally evenly distributed and located across the image (Figure 6). The selected check point positions may be located with reference to the positions of the GCPs used to correct the imagery in order to ensure that the two sets of points are independent (CPs should not be close to the GCPs).
The location or the distribution of the checkpoints is also specified in NSSDA and JRC guidelines. These Standards assume that the area to be evaluated is a rectangle and is divided into four quads and a diagonal is to be established across the area. At least $20 \%$ of the points should lie in each quarter whereas the optimum distance between points (is related to the diagonal distance of the area (1/10th of the diagonal length).

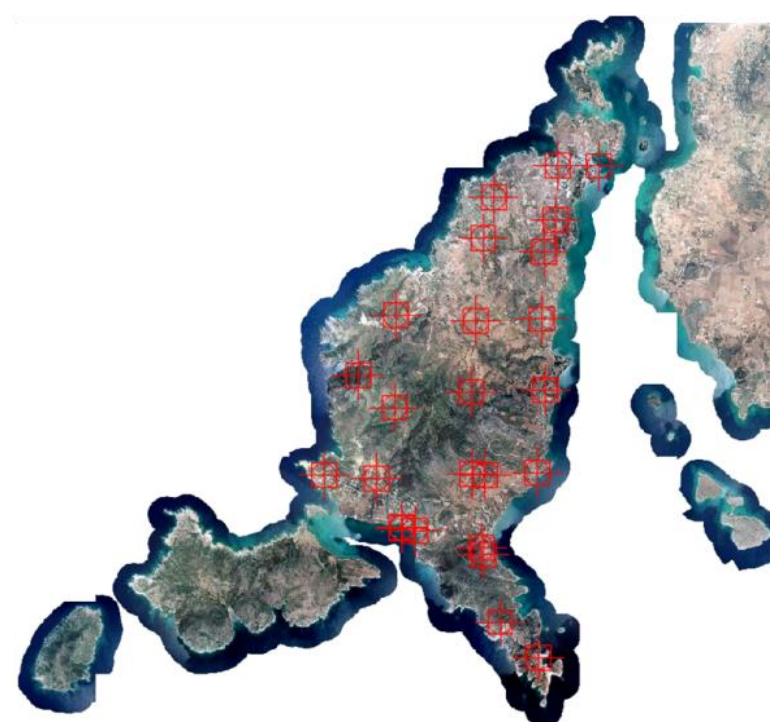

Figure 6. Checkpoints distribution on control dataset

4.2.4 Measurements - Results: 25 checkpoints were measured and used for the Pleiades 1B dataset and 26 checkpoints were used for the GeoEye-1 and LSO data scattered into areas with different terrain relief and land cover (Figure 5).

\begin{tabular}{|c|c|c|c|c|c|c|c|c|}
\hline & \multicolumn{2}{|c|}{ VLSO (Control Dataset) } & \multicolumn{2}{c|}{ PLEIADES B1 } & \multicolumn{2}{c|}{ GEOEYE 1 } & \multicolumn{2}{c|}{ LSO } \\
\hline $\mathbf{A} / \mathbf{A}$ & $\mathbf{X}(\mathbf{m})$ & $\mathbf{Y}(\mathbf{m})$ & $\mathbf{d X}(\mathbf{m})$ & $\mathbf{d Y}(\mathbf{m})$ & $\mathbf{d X}(\mathbf{m})$ & $\mathbf{d Y}(\mathbf{m})$ & $\mathbf{d X}(\mathbf{m})$ & $\mathbf{d Y}(\mathbf{m})$ \\
\hline $\mathbf{1}$ & 596781,77 & 4100183,44 & $-0,69$ & $-0,28$ & $-0,56$ & 1,12 & 0,24 & 0,47 \\
\hline $\mathbf{2}$ & 595845,18 & 4100188,57 & $-0,73$ & $-0,02$ & $-0,02$ & 0,89 & $-0,39$ & 0,35 \\
\hline $\mathbf{3}$ & 595813,14 & 4098975,40 & $-0,05$ & $-0,17$ & $-0,18$ & 1,24 & 0,29 & 0,40 \\
\hline $\mathbf{4}$ & 594419,04 & 4099474,79 & 0,51 & $-0,09$ & $-0,24$ & 0,46 & 0,52 & 1,09 \\
\hline $\mathbf{5}$ & 595546,93 & 4098254,51 & $-0,37$ & 0,23 & $-0,24$ & 0,79 & 0,84 & 0,86 \\
\hline $\mathbf{6}$ & 594187,66 & 4098555,68 & $-0,23$ & $-0,78$ & $-0,34$ & 0,08 & $-0,19$ & $-0,10$ \\
\hline $\mathbf{7}$ & 595494,50 & 4096742,60 & $-0,45$ & 0,49 & $-0,58$ & 1,07 & $-0,29$ & 0,87 \\
\hline $\mathbf{8}$ & 595406,89 & 4096729,21 & $-0,28$ & $-0,04$ & 0,58 & 1,52 & 0,31 & 0,41 \\
\hline $\mathbf{9}$ & 594027,42 & 4096706,90 & $-0,63$ & $-0,70$ & 0,28 & 0,27 & 0,11 & 0,26 \\
\hline $\mathbf{1 0}$ & 592307,54 & 4096692,48 & $-0,09$ & $-0,72$ & $-0,14$ & 1,00 & $-0,02$ & 0,89 \\
\hline $\mathbf{1 1}$ & 592229,01 & 4096831,51 & $-0,50$ & $-0,68$ & 0,00 & 1,07 & 0,16 & 0,81 \\
\hline $\mathbf{1 2}$ & 595570,94 & 4095148,65 & $-0,65$ & 0,15 & $-0,11$ & $-0,91$ & $-0,57$ & 1,12 \\
\hline $\mathbf{1 3}$ & 593924,05 & 4095109,99 & 0,39 & $-0,51$ & $-0,66$ & 0,33 & $-0,36$ & 0,91 \\
\hline $\mathbf{1 4}$ & 592201,41 & 4094754,63 & $-0,38$ & 0,04 & $-0,44$ & 0,73 & $-0,19$ & 0,57 \\
\hline $\mathbf{1 5}$ & 591581,53 & 4095387,22 & $-0,51$ & $-0,6$ & $-0,35$ & 0,78 & $-0,39$ & 0,64 \\
\hline $\mathbf{1 6}$ & 591386,71 & 4095474,95 & 0,47 & $-0,14$ & 0,86 & 1,04 & 0,02 & 0,74 \\
\hline $\mathbf{1 7}$ & 595389,69 & 4093290,39 & - & - & 0,08 & $-1,31$ & $-0,36$ & 0,07 \\
\hline $\mathbf{1 8}$ & 593939,63 & 4093262,95 & 0,69 & $-0,56$ & 0,73 & 0,01 & $-0,55$ & 0,16 \\
\hline $\mathbf{1 9}$ & 591828,22 & 4093270,46 & $-0,77$ & $-0,55$ & $-1,48$ & $-0,46$ & $-0,68$ & $-0,20$ \\
\hline $\mathbf{2 0}$ & 591811,63 & 4093172,45 & $-0,51$ & $-0,2$ & $-0,57$ & 0,41 & $-0,40$ & 0,20 \\
\hline $\mathbf{2 1}$ & 590625,15 & 4093229,84 & 0,18 & $-0,91$ & $-0,37$ & 0,95 & $-0,45$ & 0,70 \\
\hline $\mathbf{2 2}$ & 592663,35 & 4091993,05 & 0,11 & 0,26 & $-0,22$ & 0,71 & $-0,14$ & 0,54 \\
\hline $\mathbf{2 3}$ & 592370,47 & 4092052,04 & 0,09 & 0,49 & $-0,13$ & 0,98 & $-0,12$ & 0,36 \\
\hline $\mathbf{2 4}$ & 592357,15 & 4092062,40 & 0,22 & 0,04 & $-0,08$ & 0,68 & $-0,71$ & 0,47 \\
\hline $\mathbf{2 5}$ & 594197,98 & 4091451,92 & $-0,28$ & $-0,28$ & $-0,27$ & 0,18 & $-0,06$ & $-0,34$ \\
\hline $\mathbf{2 6}$ & 595326,84 & 4089050,41 & $-0,16$ & 0,61 & $-0,44$ & 0,29 & $-0,05$ & 0,04 \\
\hline
\end{tabular}

Table 1. Measured coordinates for GeoEye-1 and Pleiades 1B orthoimagery.

The differences $\mathrm{dX}, \mathrm{Y}$, are computed as $\boldsymbol{d} \boldsymbol{X}, \boldsymbol{Y}=\boldsymbol{X}, \boldsymbol{Y}_{\text {data }}-\boldsymbol{X}, \boldsymbol{Y}_{\text {check }}$ 
It is noted that for Pleiades $1 \mathrm{~B}$ was determined 1 checkpoint less due to the clouds over the area of the point. The coordinates of these checkpoints were determined on the control dataset, the aerial imagery orthomosaic (VLSO) provided from Hellenic National Cadastre \& Mapping Agency S.A. (NCMA S.A) $(0.25 \mathrm{~m}$ GSD). In Table 1 are presented the measured coordinates of the checkpoints on the control dataset and the differences between those and the measured coordinates in the test datasets.

The results are presented with a statistical analysis and they are evaluated in order to present the merits and demerits of the imaging sensors involved. To this direction the Standard Deviation $(\sigma)$ or sigma are computed as an indicator of how well the measurements fit each other and a measure of precision. In addition, the Root Mean Squares Error (RMSE) is computed for Northing and Easting coordinates. It is assumed that errors in the spatial data have random behavior and that systematic errors have been eliminated as best as possible

$$
\begin{aligned}
& S t D E V=\sqrt{\sum \frac{d^{2}}{n-1}} \\
& R M S E_{X}=\sqrt{\sum_{i=1}^{n}\left(X_{\text {data }}-X_{\text {check }}\right)^{2}} \\
& R M S E_{Y}=\sqrt{\sum_{i=1}^{n}\left(Y_{\text {data }}-Y_{\text {check }}\right)^{2}} \\
& R M S E_{X Y}=\sqrt{\left(R M S E_{X}\right)^{2}-\left(R M S E_{Y}\right)^{2}} \\
& d=\text { the deviation } \\
& n=\text { the number of check points } \\
& X, Y_{\text {check }}=\text { the check points coordinates } \\
& \text { measured on control dataset and } \\
& X, Y_{\text {data }}=\text { the points coordinates measured } \\
& \text { on test dataset }
\end{aligned}
$$

where $\quad d=$ the deviation

\begin{tabular}{|l|c|c|c|}
\hline & \multicolumn{3}{|c|}{ RESIDUALS } \\
\cline { 2 - 4 } & \multicolumn{3}{|c|}{$\mathbf{X}$} \\
\hline & Pleiades B1 & Geoeye-1 & LSO \\
\hline Chck Pts & 25 & 26 & 26 \\
\hline StDEV(m) $^{*}$ & 0.374 & 0.388 & 0.467 \\
\hline RMSE $_{\mathbf{X}}(\mathbf{m})$ & 0.389 & 0.607 & 0.495 \\
\hline & \multicolumn{3}{|c|}{$\mathbf{Y}$} \\
\hline & Pleiades B1 & Geoeye-1 & LSO \\
\hline Check Pts & 25 & 26 & 26 \\
\hline StDEV (m) & 0.658 & 0.415 & 0.423 \\
\hline RMSE $_{\mathbf{Y}}(\mathbf{m})$ & 0.838 & 0.453 & 0.465 \\
\hline
\end{tabular}

Table 2. Resulting residuals of the measured checkpoints

As observed, in aerial imagery LSO and Geoeye-1 orthomosaic, the Standard Deviation and $\mathrm{RMSE}_{X, Y}$ differ indicating a systematic error in $\mathrm{Y}$ axis. Thus, a bias removal procedure was applied in order to evaluate orthoimages objectively and accurately.

The BIAS is estimated by the formula:

$$
B I A S=\sqrt{R M S E_{X Y}^{2}-\sigma^{2}}
$$

where

$\sigma=$ is the random error

\begin{tabular}{|c|c|c|c|c|c|c|}
\hline Data Src & \multicolumn{2}{|c|}{ Pleiades B1 } & \multicolumn{2}{c|}{ Geoeye-1 } & \multicolumn{2}{c|}{ LSO } \\
\hline RMSx,y - stdev & 0,038 & 0,042 & 0,028 & 0,179 & 0,016 & 0,219 \\
\hline Linear Bias & $\mathbf{0 , 1 8 1}$ & $\mathbf{0 , 1 9 3}$ & $\mathbf{0 , 1 6 4}$ & $\mathbf{0 , 5 1 8}$ & $\mathbf{0 , 1 1 0}$ & $\mathbf{0 , 4 6 7}$ \\
\hline Circular Bias & \multicolumn{3}{|c|}{0,265} & \multicolumn{2}{c|}{0,543} & \multicolumn{2}{c|}{0,479} \\
\hline \multicolumn{3}{|c|}{ Table 3. Bias calculations }
\end{tabular}

\begin{tabular}{|c|c|c|c|}
\hline & Pleiades B1 & Geoeye-1 & LSO \\
\hline RMSE $_{\mathbf{X}}(\mathbf{m})$ & 0.522 & 0.381 & 0.578 \\
\hline RMSE $_{\mathbf{Y}}(\mathbf{m})$ & 0.646 & 0.453 & 0.465 \\
\hline & \multicolumn{3}{|c|}{ Horizontal RESIDUALS } \\
\hline & Pleiades B1 & Geoeye-1 & LSO \\
\hline RMSE $_{\mathbf{X Y}}(\mathbf{m})$ & 0.646 & 0.866 & 0.649 \\
\hline
\end{tabular}

Table 4. RMSEs and horizontal residuals afters bias removal

Geoeye-1 data have the bigger $\mathrm{RMSE}_{\mathrm{XY}}$ possibly due to bad image quality leading to erroneous GCPs measurements on the images. Pleiades 1B orthoimage has almost the same external accuracy as the orthomosaic from aerial imagery (LSO from NCMA S.A).

\section{CONCLUSION}

The main aim of this paper was to assess the geometric and radiometric quality of orthorectified high resolution satellite imagery from Pleiades-1B satellites through a comparative evaluation of their quantitative and qualitative properties. In addition, the advantages and limits of the Pleiades Imaging for producing Large Scale Orthophotos (LSO) are investigated. The visual assessment of the orthoimagery revealed that Pleiades 1B orthoimagery is especially promising presenting much more information and clearer forms. On the contrary, abrupt changes of brightness and contrast and high radiometric saturation levels were observed on the GeoEye-1 orthomosaic. The geometric evaluation reveals that the used LSO and Geoeye-1 orthomosaic suffer from a systematic error in $\mathrm{Y}$ axis. In addition, Pleiades $1 \mathrm{~B}$ and LSO imagery have almost the same external accuracy.

Both radiometric and accuracy test results show that Pleiades 1B orthoimage has almost the same absolute accuracy as the orthomosaic from aerial imagery (LSO from Hellenic National Cadastre \& Mapping Agency S.A.). Hence, it could easily replace aerial imagery, when it comes to orthoimage production. Furthermore, all data are adequate for producing LSO for mapping and GIS, according to JRC and NSSDA accuracy standards. The results also serve for a critical approach for the usability and cost efficiency of satellite imagery for the production of LSO.

\section{ACKNOWLEDGEMENTS}

The research leading to these results has been supported by European Union funds and National funds (GSRT) from Greece and EU under the project JASON: Joint synergistic and integrated use of eArth obServation, navigation and commuNication technologies for enhanced border security funded under the cooperation framework. The authors would also like to thank Astrium GEO-Information Services which provided the Pléiades triplet for research and investigation purposes. Finally, the contribution of NCMA S.A, through the provision of the orthophoto and DEM, produced by aerial photography is acknowledged.

\section{REFERENCES}

Aguilar, M. A., Agüera, F., Aguilar, F. J., and Carvajal, F., 2008. Geometric accuracy assessment of the orthorectification 
process from very high resolution satellite imagery for Common Agricultural Policy purposes. International journal of remote sensing, 29(24), pp. 7181-7197.

Astrium, 2012. Pléiades Imagery User Guide. October 2012v2.0.

Chavez, P. and Bowell, J., 1988. "Comparison of the spectral information content of Landsat thematic mapper and spot for three different sites in the Phoenix, Arizona region," Photogramm. Eng. Remote Sens., vol. 54, no. 12, pp. 16991708 .

Cheng, Ph., Chaapel, C., 2008. Using WorldView-1 stereo data with or without ground control points. GEOinformatics 11 (7), pp. 34-39.

Crespi, M., and De Vendictis, L., 2009. A procedure for high resolution satellite imagery quality assessment. Sensors, 9(5), pp. 3289-3313.

Edwards, K. and Davis, P., 1994. The use of intensity-huesaturation transformation for producing color shaded relief images, Photogramm. Eng. Remote Sens., vol. 60, no. 11, pp. 1369-1373.

Eisenbeiss, H., Baltsavias, E., Pateraki, M., and Zhang, L., 2004. Potential of IKONOS and QUICKBIRD imagery for accurate 3D-Point positioning, orthoimage and DSM generation. International Archives of the Photogrammetry, Remote Sensing and Spatial Information Sciences, 35(B3), pp. 522-528.

FGDC. 1998. Geospatial Positioning Accuracy Standards Part 3: National Standard for Spatial Data Accuracy, FGDC-STD007.3-1998 https://www.fgdc.gov/standards/projects/FGDCstandards-projects/accuracy/part3/index_html

Gleyzes, M. A., Perret, L., and Kubik, P., 2012. Pleiades system architecture and main performances. International Archives of the Photogrammetry, Remote Sensing and Spatial Information Sciences, 39, B1.

Gonzalez, R. C., and Woods, R. E., 2002. Digital image processing, 2nd. SL: Prentice Hall, 2.

Greenfeld, J., 2001. Evaluating the accuracy of Digital Orthophoto Quadrangles (DOQ) in the context of parcel-based GIS. Photogrammetric engineering and remote sensing, 67(2), pp. 199-206.

Haydn, R., Dalke, G. W., Henkel, J., and Bare, J. E., 1982. Application of the IHS color transform to the processing of multisensor data and image enhancement. In Proceedings of the International Symposium on Remote Sensing of Environment, First Thematic Conference:" Remote sensing of arid and semiarid lands", Cairo, Egypt.

Helder, D., Choi, J., 2003. On-orbit Modulation Transfer Function (MTF) measurements on QuickBird. In: Proceedings of the 2003 High Spatial Resolution Commercial Imagery Workshop, NASA/NIMA/USGS Joint Agency Commercial Imagery Evaluation Team: Reston, VA, USA.
Ioannidis, C., and Katsigiannis, A., 2006. Accuracy comparison tests on ortho-rectified high resolution satellite images. Revue française de photogrammétrie et de télédétection, (184), 95-100.

Ioannou, M. T., and Georgopoulos, A., 2013.Evaluating large scale orthophotos derived from high resolution satellite imagery. In First International Conference on Remote Sensing and Geoinformation of Environment. International Society for Optics and Photonics, pp. 879515-879515.

Jacobsen, K.,2011. Characteristics of very high resolution optical satellites for topographic mapping. International Archives of the Photogrammetry, Remote Sensing and Spatial Information Sciences, XXXVIII (4/W19), on CDROM.

Kapnias, D., Milenov, P., and Kay, S. 2008. Guidelines for Best practice and quality checking of ortho imagery. Joint Research Centre, (3.0).

Kohm, K., 2004. Modulation transfer function measurement method and results for the OrbView-3 high resolution imaging satellite. International Archives of the Photogrammetry, Remote Sensing and Spatial Information Sciences, 35 (B1), pp. 7-12.

Leger, D., Viallefont, F., Deliot, P., Valorge, C., 2004. On-orbit MTF assessment of satellite cameras. In: Morain, S.A., Budge, A.M. (Eds.), Post-Launch Calibration of Satellite Sensors. Taylor and Francis Group, London, UK, pp. 67-76.

Niu, X., Wang, J., Di, K., Lee, J-D., Li R., 2004. Geometric modelling and photogrammetric processing of high-resolution satellite imagery. In: The International Archives of the Photogrammetry, Remote Sensing and Spatial Information Sciences, Istanbul, Turkey, Vol. XXXV, Part B4, pp. 689-694.

Padwick, C., Deskevich, M. I. C. H. A. E. L., Pacifici, F., and Smallwood, S., 2010. WorldView-2 pan-sharpening. Proc. American Society for Photogrammetry and Remote Sensing, 13.

Palsson, F., Sveinsson, J. R., Benediktsson, J. A., and Aanaes, H., 2012. Classification of pansharpened urban satellite images. Selected Topics in Applied Earth Observations and Remote Sensing, IEEE Journal of, 5(1), pp. 281-297.

Poli, D., Remondino, F., Angiuli, E., and Agugiaro, G., 2014. Radiometric and geometric evaluation of GeoEye-1, WorldView-2 and Pléiades-1A stereo images for 3D information extraction. ISPRS Journal of Photogrammetry and Remote Sensing.

Tu, T. M., Huang, P. S., Hung, C. L., and Chang, C. P., 2004. A fast intensity-hue-saturation fusion technique with spectral adjustment for IKONOS imagery. Geoscience and Remote Sensing Letters, IEEE, 1(4), pp. 309-312.

Carper, W. J., Lillesand, T. M., and Kiefer, P. W., The use of intensity-hue-saturation transformations for merging spot panchromatic and multispectral image data, Photogramm. Eng. Remote Sens., vol. 56, no. 4, pp. 459-467.

Zhou, G., Li, R., 2000. Accuracy evaluation of ground points from IKONOS high-resolution satellite imagery. Photogrammetric Engineering and Remote Sensing, 66(9), pp. 1103-1112. 